(C) 1983 IEEE. Personal use of this material is permitted. However, permission to reprint/republish this material for advertising or promotional purposes or for creating new collective works for resale or redistribution to servers

or lists, or to reuse any copyrighted component of this work in other works must be obtained from the IEEE.

IEEE Transactions on Nuclear Science, Vol. NS-30, No. 4, August 1983

WIGGLERS FOR CONTROL OF BEAM CHARACTERISTICS IN LEP

\author{
J.M. Jowett and T.M. Taylor \\ LEP Division \\ CERN
}

1211 Geneva 23, Switzerland

Summary

Eight three-block dipole wigglers will be used in LEP to maximize luminosity and control other beam parameters such as emittance, damping rates at injection, energy spread and polarization. The excitation law for constant emittance (chosen, for example, to fill the dynamic aperture) and damping partition numbers is given. In contrast to conventional wigglers where orbit perturbation is avoided by means of a series of equal fields of alternate polarity, the LEP wigglers will use unequal fields and pole lengths. This allows one to retain a high potential asymptotic polarisation level together with an increased polarisation rate. The choice of the degree of field asymmetry is imposed by limitations on the transverse dimensions of the magnets and field levels, by polarisation requirements and by cost. A practical design of the proposed wiggler is presented.

\section{Emittance Control}

In an electron-positron storage ring with fixed optics, the emittances and energy spread of the beam are uniquely determined by the operating energy. Putting in a dipole wiggler makes it possible to have larger energy spreads and emittances than these "natural" values 1 . When the possibility of increasing the coupled horizontal emittance, $\varepsilon_{x c}$, by reducing the damping partition number, $J_{x}$, is exhausted, a wiggler allows the storage of larger currents before reaching the beambeam 1 imit (assumed to correspond to a definite value, e.g. $\xi=0.03$, of the linear tune-shift parameter). Together with the requirement of keeping the occupied region of phase space sufficiently within the dynamic (or physical) aperture, this leads to the prescription of constant $\varepsilon_{x c}=\hat{\varepsilon}_{x c}$ for maximum stored current at energy $E<\hat{E}$. This is the energy at which the dynamic aperture constraints are just fulfilled with the lowest tolerable value of $\mathrm{J}_{\mathrm{x}}(0.5$, for example) and the wiggler unexcited. In the high-luminosity configurations of machines like LEP, the dynamic aperture is generally smaller than the physical aperture for $\mathrm{E}<\hat{\mathrm{E}}$. With standard notations:

$$
\varepsilon_{x c}=\frac{1}{1+\kappa^{2}} \frac{55}{32 \sqrt{3}}\left(\frac{\hbar}{m c}\right)\left(\frac{E}{m c^{2}}\right)^{2} \frac{I_{5}^{0}+I_{5}^{W}}{J_{x}\left(I_{2}^{0}+I_{2}^{W}\right)},
$$

where $I_{i}^{5}$ and $I_{i}^{W}(i=1, \ldots, 5)$ are the contributions of the lattice and the wiggler to the synchrotron integrals, respectively.

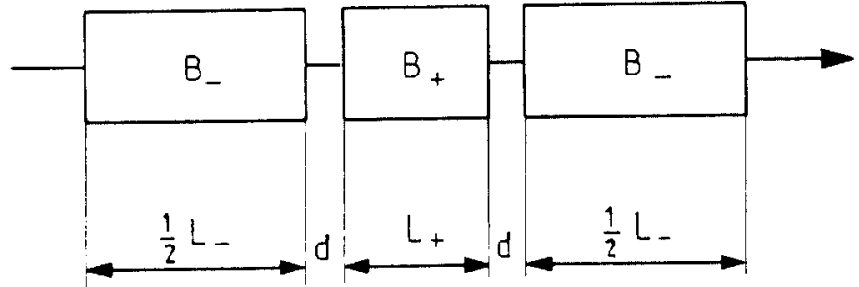

Fig. 1 Model used for calculations.
With $\mathrm{N}_{\mathrm{s}}$ of the wiggler installation shown schematically in Fig. 1 , and setting $r=L_{-} / L_{+}$:

$$
\begin{aligned}
& I_{2}^{W}=N_{s} L_{-}(1+r) G_{-}^{2}, \quad \text { and } \\
& I_{5}^{W}=N_{S} L_{-}\left(1+r^{2}\right)\langle W\rangle_{W}\left|G_{-}\right|^{3},
\end{aligned}
$$

where $G_{-}=G_{+} / r$ is the inverse of the bending radius in the outer ' - ' blocks of the wiggler. The function

$$
w=\left[D_{x}^{2}+\left(B_{x} D_{x}^{\prime}-\frac{1}{2} \beta_{x}^{\prime} D_{x}\right)^{2}\right] / \beta_{x}
$$

is changed by the excitation of the wiggler but this can be neglected in the cases of interest.

Neglecting the energy lost by synchroton radiation in the wiggler in comparison with that in the rest of the machine allows one to solve the equation for constant emittance and gives:

$$
B_{-}(E) \simeq \pm \frac{1}{e c}\left[\frac{I_{5}^{0}}{N_{s}\left(1+r^{2}\right) L_{-}\langle W\rangle_{w}}\right]^{\frac{1}{3}} \widetilde{E}, B_{+}=-r B_{-},
$$

where $\widetilde{E}$ is the geometric mean of $\hat{E}-E, E$ and $\hat{E}+E$. This excitation provides maximum luminosity with colliding beams and need not be followed during ramping. Injection can also be facilitated by using a high field level to increase damping rates.

The solid curve in Fig. 2 shows how the required wiggler field varies as a function of energy. It is interesting to note that the highest wiggler field occurs at $\mathrm{E}=\hat{\mathrm{E}} / \sqrt{3}$.

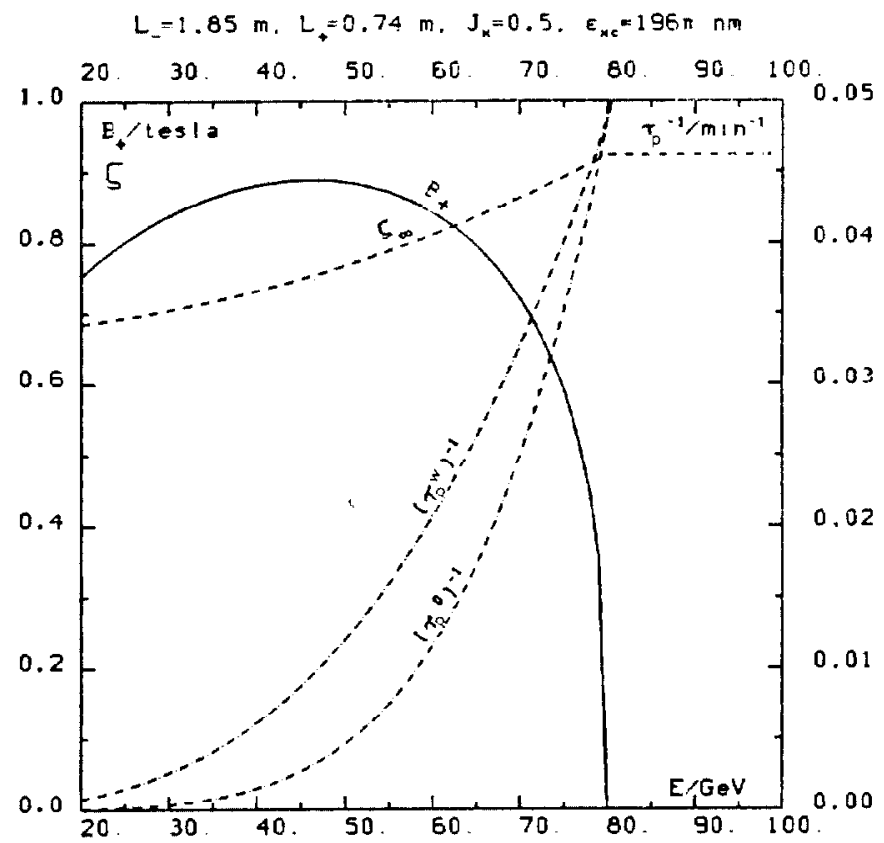

Fig. 2 Typical curves of required wiggler excitation and corresponding polarization 


\section{Polarization}

It is well known ${ }^{2}$ that the polarization rate is increased by a wiggler. Using our notation, the enhancement factor with constant emittance $c$ an be written

$$
\left(\frac{\tau_{\mathrm{p}}^{\mathrm{w}}}{\tau_{\mathrm{p}}^{0}}\right)^{-1} \simeq 1+\frac{\left\langle\mathrm{W}_{0}\right.}{\left\langle\mathrm{W}_{\mathrm{w}}\right.}\left[\left(\frac{\hat{\mathrm{E}}}{\mathrm{E}}\right)^{2}-1\right] \text {, }
$$

$<>_{0}$ being an average over the main bending magnets. However, it is also well known that any reverse bending reduces the asymptotic polarization level (neglecting all depolarizing effects)

$$
\tau_{\infty}=\frac{8}{5 \sqrt{3}} \int G(s)^{3} \mathrm{ds} / \int|G(s)|^{3} \mathrm{ds} .
$$

With our wiggler and constant $E_{\mathbf{X c}}$ :

$$
\zeta_{\infty}=\frac{8}{5 \sqrt{3}}\left\{1-\frac{2 r^{2}}{\left(1+r^{2}\right)} \frac{\left(\hat{E}^{2}-E^{2}\right)}{\left[E^{2}\left(\langle W\rangle{ }_{w} /\langle W\rangle_{0}-1\right)+\hat{E}^{2}\right]}\right\}
$$

provided the '-' sign has been chosen in the expression for $B_{-}(E)$. Thus, the bigger $r$ is, the less the maximum polarization is reduced, provided the field in the ' + ' block points in the same direction as the field of the main bending magnets. For $r=1$ or $B_{-}>0, \zeta_{\infty}$ is severely reduced.

\section{Choice of Wiggler Geometry}

The maximum values of the wiggler fields $B_{+}$and $B_{-}$ and their ratio $=$ have been chosen to provide sufficient capacity for emittance control in all optics of both the $60^{\circ}$ and $90^{\circ}$ phase advance versions of LEP, and to retain the possibility of having useful degrees and rates of polarization. Further constraints come from considerations of synchrotron radiation, available space and, of course, the cost of the units.

The wiggler system will consist of eight identical magnets, each having $\mathrm{r}=2.5, \mathrm{~L}_{+}=0.74 \mathrm{~m}$ and $\mathrm{B}_{+} \leq 1 \mathrm{~T}$, and its physical design is shown in Fig. 3 . The magnet is made in two halves separated by four nonmagnetic pillars, each half consisting of three poles fixed to a rigid steel beam (yoke). Excitation is provided by aluminium coils around the poles. Whereas the coils around the centre poles consist of a main, watercooled winding only, the end coils incorporate main and auxiliary windings. The latter serve to trim the field to give a rigorously zero field integral at all current levels. All the main windings are connected in series, as are the auxiliary windings, to give one high- and one low-current circuit $(600 \mathrm{~A}$ and $75 \mathrm{~A})$. Shims are placed at the pole edges to ensure that the field intcgral is zero across the useful aperture ( $\pm 60 \mathrm{~mm}$ ) of the magnet. Yoke and poles are 1 aminated to avoid field quality problems due to eddy currents, when the field is changed with stored beams present. The poles are $280 \mathrm{~mm}$ wide, and the gap is $100 \mathrm{~mm}$ high.

The flux configuration in this magnet is such that it is necessary to use fully three-dimensional field computation codes to determine steel and coil geometries. This has been done, and the detailed magnetic design will be described elsewhere ${ }^{3}$. The computed maximum vertical field along the beam path over half the magnet's length is shown in Fig. 4. The total excitation which is required for centre and end coils in order to obtain a zero field integral is shown in Fig. 5.

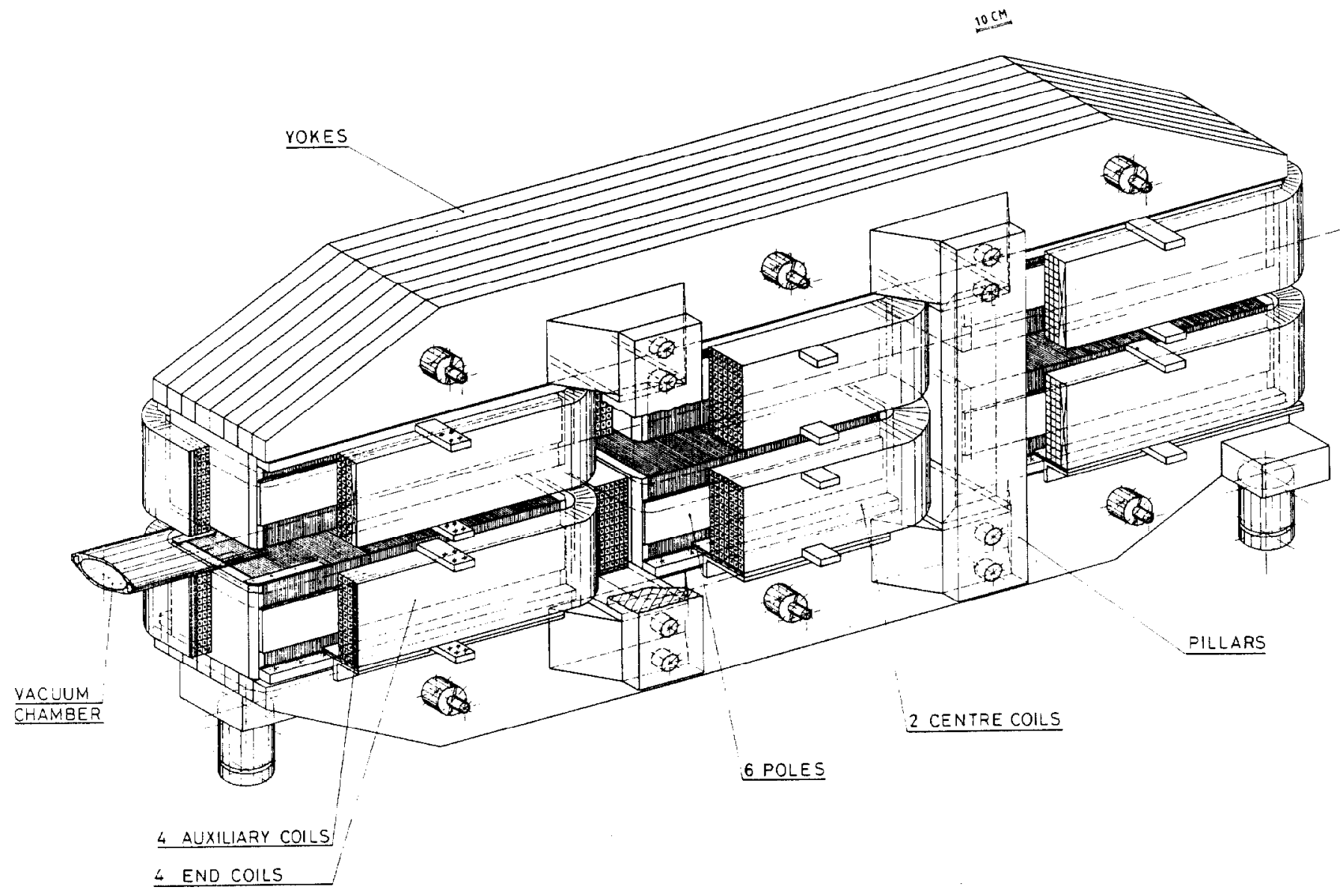

Fig. 3 Proposed LEP wiggler magnet 


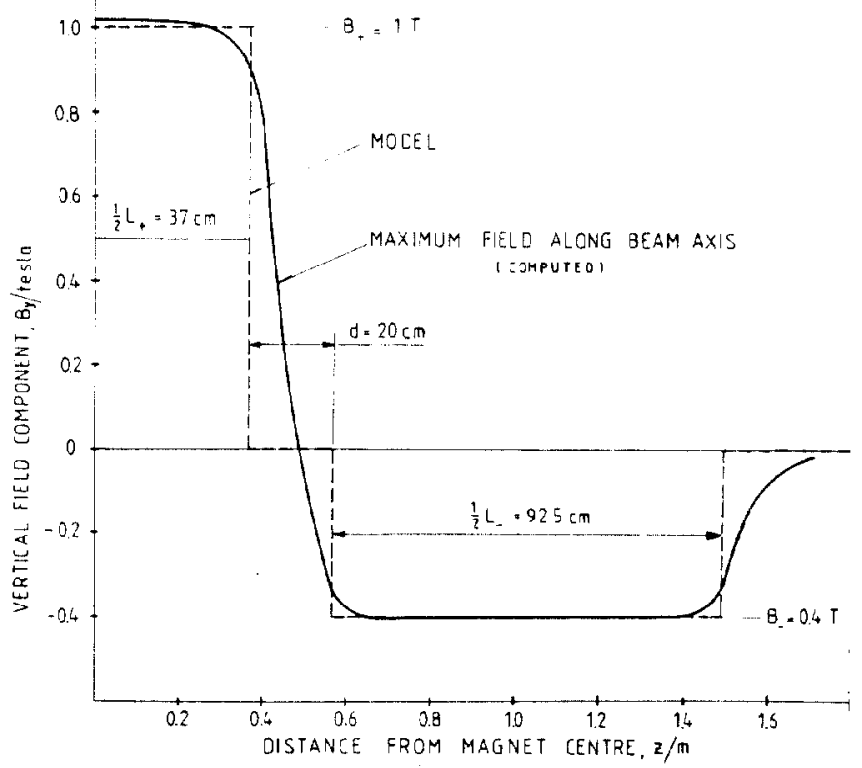

Fig. 4 Computed field over half the length of the magnet.

\section{Optical Properties}

The trajectory of the beam through the wiggler is such that edge focusing will occur at the passages from - to + and from + to - fields. Whereas the effects are self-compensating in the horizontal plane, in the vertical plane they add, and the wiggler acts as a thin focusing lens. In the worst case, i.e. at injection energy (20 GeV) and with the wiggler fully excited, the focal length of this lens is $4300 \mathrm{~m}$ : this gives $r i s e$ to a slight mismatch of the vertical beta function and a total vertical Q-shift of about 0.01 for the ensemble of eight magnets. Il is foreseen to compensate for this either by applying a general correction, or by matching, using nearby quadrupoles. The latter method is facilitated by the fact that, as the wigglers will be installed at the lattice end of the dispersion suppressor, the closest quadrupoles are individually controlled, independent of the lattice strings.

The maximum sagitta in the wiggler, occurring at injection, is estimated to be $4.7 \mathrm{~mm}$. In order to minimize the required horizontal aperture, the wiggler mag-

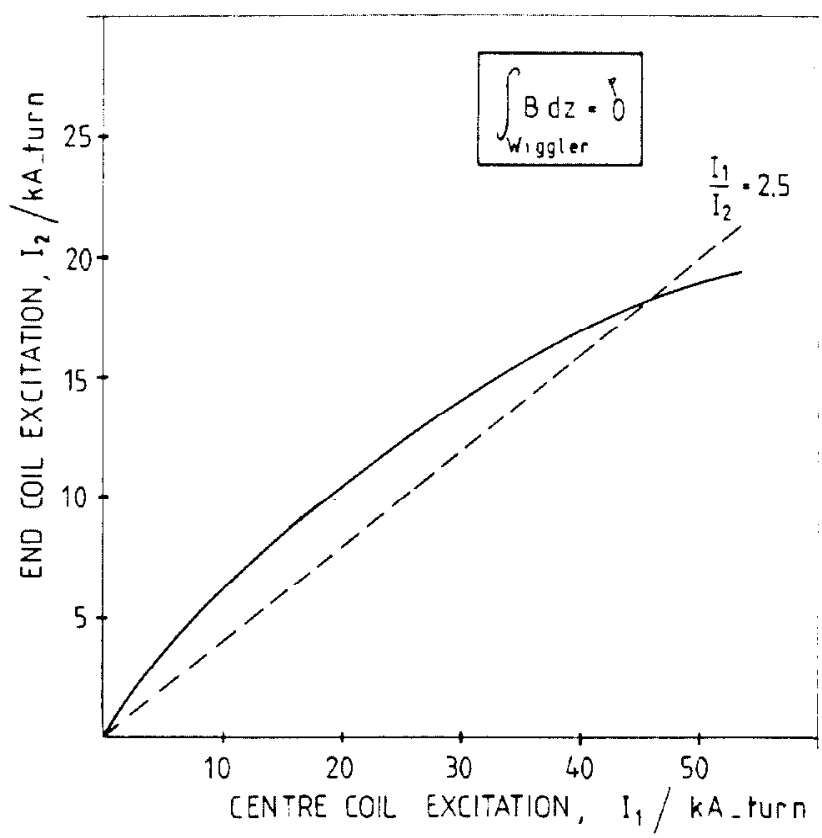

Fig. 5 End coil vs, centre coil excitation for no net effect on the closed orbit.

nets will be located near to vertically focusing quadrupoles, i.e. where $B_{x}$ is small. Such a location of the magnet is also favourable for spreading the synchrotron radiation in the vertical plane.

\section{References}

1. M. Bassetti, LNF Int. Memo T-66 (April 1971). LSR Working Group, Div. Report CERN ISR-AS/74-66 (October 1974).

J.M. Paterson, J.R. Rees and H. Wiedemann, Report SPEAR-186, PEP-125 (July 1975).

A. Hutton, Particle Accelerators, 7, p. 177 (1976).

2. A.M. Kondratenko and B.W. Montague, Div. Report CERN ISR-TH/80-38 (1980).

3. T.M. Taylor and T. Tortschanoff, Paper to be submitted for publication to the $8 \mathrm{th}$ Int. Conference on Magnet Technology (MT-8), Grenoble (1983). 